\title{
Sturmian maximizing measures for the piecewise-linear cosine family
}

\author{
V. Anagnostopoulou, K. Díaz-Ordaz, O. Jenkinson \& C. Richard
}

\begin{abstract}
Let $T$ be the angle-doubling map on the circle $\mathbb{T}$, and consider the 1parameter family of piecewise-linear cosine functions $f_{\theta}: \mathbb{T} \rightarrow \mathbb{R}$, defined by $f_{\theta}(x)=$ $1-4 d_{\mathbb{T}}(x, \theta)$. We identify the maximizing $T$-invariant measures for this family: for each $\theta$ the $f_{\theta}$-maximizing measure is unique and Sturmian (i.e. with support contained in some closed semi-circle). For rational $p / q$, we give an explicit formula for the set of functions in the family whose maximizing measure is the Sturmian measure of rotation number $p / q$. This allows us to analyse the variation with $\theta$ of the maximum ergodic average for $f_{\theta}$.
\end{abstract}

\section{Introduction}

For $T: X \rightarrow X$ a continuous map on a compact metric space $X$, let $\mathcal{M}$ denote the (weak-* compact) set of $T$-invariant Borel probability measures on $X$. Given a continuous function $f: X \rightarrow \mathbb{R}$, a measure $\mu \in \mathcal{M}$ is said to be maximizing for $f$ if

$$
\int f d \mu=\max _{m \in \mathcal{M}} \int f d m,
$$

i.e. if $\mu$ attains the largest possible ergodic average for $f$.

This ergodic optimization problem, concerning the maximizing measure(s) (and corresponding maximum ergodic average) for a given triple $(X, T, f)$, has been the focus of considerable recent attention (see e.g. [29] for an overview). Most of this work has concerned theoretical aspects of the subject, including abstract information on the nature of maximizing measures $[8,10,11,13,19,30,36,37,39,40,41,42,44$, $47,48,49,51]$, approximation of maximizing measures $[9,15,18]$, connections with thermodynamic formalism $[12,17,27,28,33,35,38,46]$, and connections with partial orders on $\mathcal{M}[2,31,32,34]$.

Comparatively little work has focused on the concrete problem of precisely identifying the maximizing measure(s) for specific triples $(X, T, f)$. One reason for the relative lack of progress in this area stems from the intrinsic difficulty of the problem: for interesting $T$ (e.g. hyperbolic maps) the set $\mathcal{M}$ is large (an infinite-dimensional simplex), and the bona fide maximizing measure(s) can be approximated arbitrarily well by 'almost maximizing' measures, thus complicating the task of identifying (even conjecturally) the maximizing measure(s).

Nevertheless, much of the initial impetus behind the development of ergodic optimization revolved around specific choices of $(X, T)$, in particular the archetypal hyperbolic map $T(x)=2 x(\bmod 1)$ on the circle $X=\mathbb{T}=\mathbb{R} / \mathbb{Z}$. For this choice of $(X, T)$, 
maximizing measures for various functions $f$ were identified ${ }^{1}$, notably $f(x)= \pm \cos 2 \pi x$ and $f(x)=\sin 2 \pi x$ in [20], and the more general cosine family $g_{\theta}(x)=\cos 2 \pi(x-\theta)$ in $[\mathbf{2 4}, \mathbf{2 5}, \mathbf{2 6}]$. The maximizing measures for the family $\left(g_{\theta}\right)_{\theta \in \mathbb{T}}$ turned out (see $[\mathbf{7}, \mathbf{2 5}, \mathbf{2 6}, \mathbf{2 7}])$ to be a certain 1 -parameter family of $\times 2$-invariant measures known as Sturmian.

One definition of Sturmian measures is that they are precisely the invariant probability measures whose support is contained in a closed semi-circle (each closed semicircle is known to support a unique invariant probability measure, see $[\mathbf{1 6}, \mathbf{2 2}, \mathbf{5 0}]$ ). Another characterization is that these supports are precisely the non-empty minimal compact $T$-invariant sets on which the dynamics is combinatorially equivalent to a rotation (i.e. the restriction of $T$ to this support can be extended to a monotone continuous self-map of $\mathbb{T}$ ). In particular, each Sturmian measure has a well-defined rotation number $\varrho \in \mathbb{T}$, and conversely, for each $\varrho \in \mathbb{T}$ there is precisely one Sturmian measure, denoted $S_{\varrho}$, of rotation number $\varrho$. The support of $S_{\varrho}$ is a periodic orbit $\mathcal{O}_{p / q}$ if $\varrho=p / q$ is rational, and a Cantor set of zero Hausdorff dimension if $\varrho$ is irrational. For example the Sturmian measures of rotation numbers $1 / 2,1 / 3,2 / 5,3 / 8$ and 5/13 are, respectively, the invariant measures supported by the periodic orbits $\operatorname{coded}^{2}$ by the strings

$$
\text { 01, 001, 00101, 00100101, 0010010100101, }
$$

while the Sturmian measure of rotation number $(3-\sqrt{5}) / 2$ is the unique invariant probability measure supported by the orbit closure of the point with base- 2 expansion

$$
0010010100100101001010010010100101 \ldots
$$

For more details on Sturmian measures, and the related notion of Sturmian sequences, see e.g. $[1,5,7,16,22,25,26,31,32,45,50]$.

In this article we return to the setting of these original articles, fixing the dynamical system to be the angle-doubling map $T(x)=2 x(\bmod 1)$, and aiming to determine precisely the maximizing measures for a certain one-parameter family of functions $\left(f_{\theta}\right)_{\theta \in \mathbb{T}}$. This family, which we call the piecewise-linear cosine family (see Figure 1), is defined by

$$
f_{\theta}(x)=1-4 d_{\mathbb{T}}(x, \theta),
$$

where $d_{\mathbb{T}}$ denotes the usual distance function on the circle $\mathbb{T}$.

Our main result identifies the maximizing measures for all functions in this family:

THEOREM 1. Each piecewise-linear cosine function has a unique maximizing measure, and this measure is Sturmian.

So we see that the phenomenon ${ }^{3}$ of Sturmian maximizing measures has a certain robustness: the family $\left(f_{\theta}\right)_{\theta \in \mathbb{T}}$ only qualitatively resembles the cosine family $g_{\theta}(x)=$ $\cos 2 \pi(x-\theta)$, yet the family of maximizing measures is identical ${ }^{4}$. Theorem 1 will be proved in Section 2 in a slightly more precise form (see Theorem 7), while in Section 3

\footnotetext{
${ }^{1}$ The results in $[\mathbf{2 0}, \mathbf{2 4}, \mathbf{2 5}, \mathbf{2 6}$ ] were largely conjectural, and made rigorous in [7].

${ }^{2}$ The coding is the standard one via base- 2 expansions, so for example 001 represents the orbit $\{1 / 7,2 / 7,4 / 7\}$.

${ }^{3}$ Clearly this phenomenon is not universal, and counterexamples are easily constructed: for example if $\mathcal{O}$ is a non-Sturmian periodic orbit $(\mathcal{O}=\{1 / 5,2 / 5,3 / 5,4 / 5\}$, say) then the maximizing measure for $f(x):=-d_{\mathbb{T}}(x, \mathcal{O})$ is obviously non-Sturmian.

${ }^{4}$ Note, however, that for particular values of $\theta$ the $f_{\theta}$-maximizing measure need not coincide with the $g_{\theta}$-maximizing measure.
} 
we investigate the variation of the maximum ergodic average $\max _{\mu \in \mathcal{M}} \int f_{\theta} d \mu$ with the parameter $\theta$, identifying the intervals on which it is monotone (see Theorem 11).

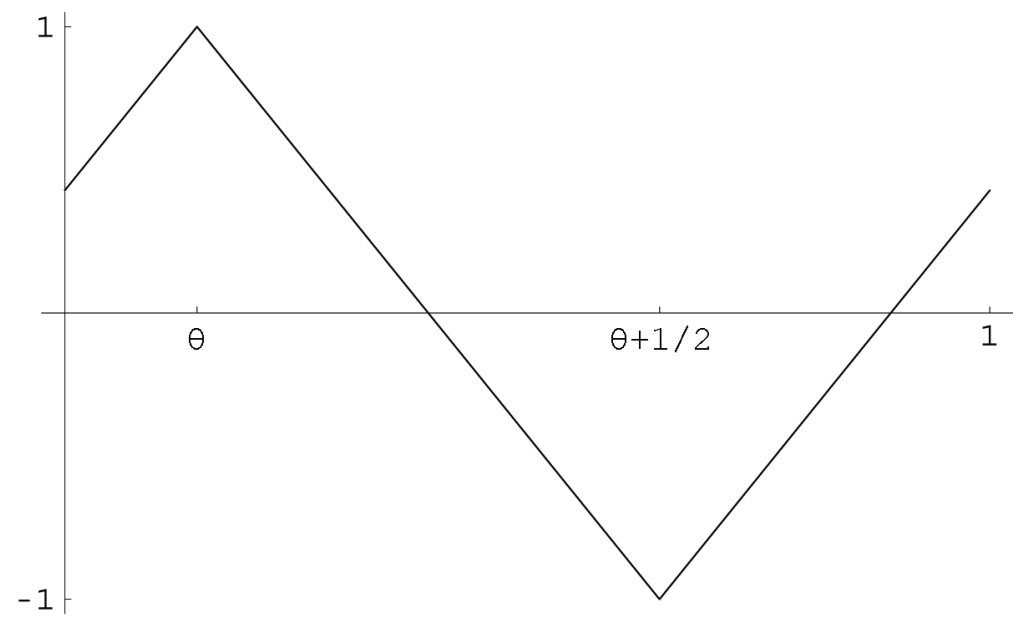

Figure 1. Piecewise-linear cosine function $f_{\theta}$.

\section{Sturmian maximizing measures}

Definition 2. For any $\gamma \in \mathbb{T}$, define $e_{\gamma}: \mathbb{T} \rightarrow \mathbb{Z}_{\geq 0} \cup\{\infty\}$ by

$$
e_{\gamma}(x)=\inf \left\{i \geq 0: T^{i}(x) \in(\gamma+1 / 2, \gamma)\right\} .
$$

Since $e_{\gamma}$ is strictly positive on $[\gamma, \gamma+1 / 2]$, there is a unique $\omega(\gamma) \in[\gamma, \gamma+1 / 2]$ such that $^{5}$

$$
\int_{\gamma}^{\omega(\gamma)} e_{\gamma}=1 / 2
$$

The map $\omega: \mathbb{T} \rightarrow \mathbb{T}$ was analysed in $[4]$, where the following was proved:

Lemma 3. $\omega: \mathbb{T} \rightarrow \mathbb{T}$ is a degree-1 homeomorphism, with $\omega^{-1}(0,1 / 8]=(-1 / 4,0]$.

Next recall the following notions from [7]:

Definition 4. Let $f: \mathbb{T} \rightarrow \mathbb{R}$ be Lipschitz, and set $\beta(f):=\max _{\mu \in \mathcal{M}} \int f d \mu$.

(i) Call a Lipschitz function $\varphi: \mathbb{T} \rightarrow \mathbb{R}$ a maximizing function ${ }^{6}$ for $f$ if, for all $x \in \mathbb{T}$,

$$
\beta(f)+\varphi(x)=\max \left((f+\varphi)\left(\frac{x}{2}\right),(f+\varphi)\left(\frac{x+1}{2}\right)\right) .
$$

(ii) Say that $f$ is Sturmian (on the closed semi-circle $[\gamma, \gamma+1 / 2]$ ) if there is a maximizing function $\varphi$ such that $(f+\varphi)(x)>(f+\varphi)(x+1 / 2)$ for all $x \in(\gamma, \gamma+1 / 2)$.

(iii) Say that $f$ is pre-Sturmian on $[\gamma, \gamma+1 / 2]$ if there exists a Lipschitz function $\varphi$ : $\mathbb{T} \rightarrow \mathbb{R}$ (not necessarily a maximizing function) such that the restriction of $f+\varphi-\varphi \circ T$ to $[\gamma, \gamma+1 / 2]$ is a constant function.

\section{REMARK 5.}

\footnotetext{
${ }^{5}$ We shall use $\int g$ to denote the Lebesgue integral of a function $g$ on the whole circle $\mathbb{T}$, and $\int_{A} g$ or $\int_{a}^{b} g$ for its Lebesgue integral over the set $A$ or interval $[a, b]$.

${ }^{6}$ Alternative terminology, motivated by notions in Lagrangian dynamics and differential geometry (see e.g. [21] for usage in this context), is that $\varphi$ is a calibrated sub-action (see e.g. [23]).
} 
(a) Since $T$ is expanding, every Lipschitz function admits a maximizing function (see [7, Lem. A]); results of this type can be traced back to the unpublished work of Conze \& Guivarc'h [20].

(b) If $f$ is Sturmian on $[\gamma, \gamma+1 / 2]$ then, for all $x \in[\gamma, \gamma+1 / 2]$,

$$
(f+\varphi)(x)=\max ((f+\varphi)(x),(f+\varphi)(x+1 / 2))=\beta(f)+\varphi(T x),
$$

so $f+\varphi-\varphi \circ T$ is equal to the constant $\beta(f)$ on $[\gamma, \gamma+1 / 2]$, and is strictly smaller than $\beta(f)$ on $(\gamma+1 / 2, \gamma)$. It follows that the Sturmian measure carried by $[\gamma, \gamma+1 / 2]$ is the unique maximizing measure for $f+\varphi-\varphi \circ T$, hence also for $f$.

LEMMA 6.

(i) If $\theta=\omega(\gamma)$, then $f_{\theta}$ is pre-Sturmian on $[\gamma, \gamma+1 / 2]$. The corresponding function $\varphi=\varphi_{\theta}$ of Definition 4 (iii) is unique up to an additive constant, with (Lebesgue almost everywhere defined) derivative $\varphi_{\theta}^{\prime}(x)=\sum_{n=1}^{\infty} 2^{-n} f_{\theta}^{\prime}\left(\tau^{n} x\right)$, where $\tau(x)$ denotes ${ }^{7}$ the unique member of $T^{-1}(x)$ lying in $C$.

(ii) If $f_{\theta}$ is pre-Sturmian on some semi-circle $[\gamma, \gamma+1 / 2]$ containing $\theta$, then $\theta=\omega(\gamma)$.

Proof. By [7, p. 503], a Lipschitz function $f$ is pre-Sturmian on $[\gamma, \gamma+1 / 2]$ if and only if $\int e_{\gamma} f^{\prime}=0$, where $f^{\prime} \in L^{\infty}(\mathbb{T})$ denotes the derivative of $f$, and in this case the function $\varphi$ of Definition 4 (iii) has derivative $\varphi^{\prime}(x)=\sum_{n=1}^{\infty} 2^{-n} f^{\prime}\left(\tau^{n} x\right)$. Since $f_{\theta}^{\prime}$ equals +4 on $(\theta-1 / 2, \theta)$, and -4 on $(\theta, \theta+1 / 2)$, the equation $\int e_{\gamma} f_{\theta}^{\prime}=0$ is equivalent to $\int_{\gamma}^{\theta} e_{\gamma}=\int_{\theta}^{\gamma+1 / 2} e_{\gamma}$, the only solutions to which are $\theta=\omega(\gamma)$, and $\theta=\omega(\gamma)+1 / 2$. Of these, only $\theta=\omega(\gamma)$ lies in $[\gamma, \gamma+1 / 2]$.

We are now able to prove the following more precise version of Theorem 1:

TheOREM 7. For each $\theta \in \mathbb{T}$, the unique $f_{\theta}$-maximizing measure is the Sturmian measure carried by the unique semi-circle $[\gamma, \gamma+1 / 2]$ satisfying $\omega(\gamma)=\theta$.

Proof. In view of Lemma 6 (ii), it suffices to show that each function $f_{\theta}$ is Sturmian on the semi-circle $[\gamma, \gamma+1 / 2]$ satisfying $\omega(\gamma)=\theta$.

Case 1: $\theta \in\left[-\frac{1}{8}, \frac{1}{8}\right]$.

By symmetry we need only consider $\theta \in\left[0, \frac{1}{8}\right]$. We claim that for each $f_{\theta}$ there exists $\gamma \in \mathbb{T}$, and a maximizing function $\varphi_{\theta}$, such that

$$
\left(f_{\theta}+\varphi_{\theta}\right)(x)>\left(f_{\theta}+\varphi_{\theta}\right)(y) \text { for all } x \in[\gamma, \gamma+1 / 2], y \in(\gamma+1 / 2, \gamma) \text {. }
$$

In particular, (4) implies that $f_{\theta}$ is Sturmian (on $[\gamma, \gamma+1 / 2]$ ).

To prove (4), first note that if $\theta=0$ then $\alpha(0)=1$ and $\varphi_{0}:=f_{0}$ is a maximizing function for $f_{0}$. Clearly $\left(f_{0}+\varphi_{0}\right)(x)=2 f_{0}(x)>2 f_{0}(y)$ for all $x \in[-1 / 4,1 / 4]$, $y \in(1 / 4,3 / 4)$, so (4) is satisfied.

Now suppose that $\theta \in(0,1 / 8]$. By Lemma 3, the map $\omega: \mathbb{T} \rightarrow \mathbb{T}$ is a homeomorphism with $\omega^{-1}(0,1 / 8]=(-1 / 4,0]$, so there exists (a unique) $\gamma \in\left(-\frac{1}{4}, 0\right]$ such that $\omega(\gamma)=\theta$. By Lemma 6 (i), $f_{\theta}$ is pre-Sturmian on $[\gamma, \gamma+1 / 2]$, i.e. there exists a Lipschitz function $\varphi_{\theta}$ such that $f_{\theta}+\varphi_{\theta}-\varphi_{\theta} \circ T \equiv 1-4 \theta$ on $[\gamma, \gamma+1 / 2]$. Indeed if $x \in(2 \gamma, 1+2 \gamma)$, Lemma 6 (i) implies

$$
\varphi_{\theta}(x)=\left(\sum_{n=1}^{\infty} f_{\theta}\left(x / 2^{n}\right)\right)-(1-4 \theta) .
$$

\footnotetext{
${ }^{7}$ The map $\tau$ is well-defined except at the point $2 \gamma=T(\gamma)=T(\gamma+1 / 2)$; in particular, each iterate $\tau^{n}$ is defined Lebesgue almost everywhere.
} 
If $x \in[2 \gamma, 0]$ and $n \geq 1$ then $x / 2^{n} \in[\gamma, 0] \subset\left[\theta-\frac{1}{2}, \theta\right]$, because $\gamma \in\left[-\frac{1}{4}, 0\right]$ and $\theta \in\left[0, \frac{1}{8}\right]$, so $f_{\theta}\left(x / 2^{n}\right)=1+4\left(\frac{x}{2^{n}}-\theta\right)$, and (5) becomes

$$
\varphi_{\theta}(x)=4 x \text { for } x \in[2 \gamma, 0] .
$$

If $i$ denotes the largest non-negative integer such that $2^{i} \theta<1+2 \gamma$, then

$$
[0,1+2 \gamma)=[0,2 \theta) \cup \ldots \cup\left[2^{i-1} \theta, 2^{i} \theta\right) \cup\left[2^{i} \theta, 1+2 \gamma\right) .
$$

If $x \in[0,2 \theta)$ then $x / 2^{n} \in[0, \theta)$ for all $n \geq 1$, and a calculation analogous to the one above gives

$$
\varphi_{\theta}(x)=4 x \text { for } x \in[0,2 \theta) .
$$

If $x \in\left[2^{j} \theta, 2^{j+1} \theta\right)$ for some $1 \leq j \leq i$, then $x / 2^{n} \in\left[\theta, \theta+\frac{1}{2}\right)$ for $1 \leq n \leq j$, while $x / 2^{n} \in[0, \theta)$ for $n \geq j+1$, so

$$
f_{\theta}\left(x / 2^{n}\right)-(1-4 \theta)= \begin{cases}8 \theta-2^{2-n} x & \text { if } 1 \leq n \leq j \\ 2^{2-n} x & \text { if } n \geq j+1\end{cases}
$$

It follows that

$$
\varphi_{\theta}(x)=8 j \theta-4\left(1-2^{1-j}\right) x \quad \text { for } x \in\left[2^{j} \theta, 2^{j+1} \theta\right) .
$$

An analogous calculation yields

$$
\varphi_{\theta}(x)=8 i \theta-4\left(1-2^{1-i}\right) x \quad \text { for } x \in\left[2^{i} \theta, 1+2 \gamma\right) .
$$

We now have an explicit description of $\varphi_{\theta}$ on

$$
\mathbb{T}=[2 \gamma, 0) \cup[0,2 \theta) \cup \ldots \cup\left[2^{i-1} \theta, 2^{i} \theta\right) \cup\left[2^{i} \theta, 1+2 \gamma\right)
$$

by (6), (7), (8), and (9). In particular, $\varphi_{\theta}$ has strictly positive derivative on $[2 \gamma, 2 \theta]$, is constant on $[2 \theta, 4 \theta]$, has strictly negative derivative on $[4 \theta, 1+2 \gamma)$, and its derivative is everywhere strictly larger than -4 . Moreover, the derivative $\varphi_{\theta}^{\prime}$ is non-increasing on $[2 \gamma, 1+2 \gamma)$, so $\varphi_{\theta}$ is concave.

Now $f_{\theta}$ has derivative equal to +4 on $(2 \gamma, \theta)$, to -4 on $\left(\theta, \theta+\frac{1}{2}\right)$, and to +4 on $\left(\theta+\frac{1}{2}, 1+2 \gamma\right)$. It follows that $f_{\theta}+\varphi_{\theta}$ has derivative +8 on $(2 \gamma, \theta)$ and derivative 0 on $(\theta, 2 \theta)$. Its derivative is strictly negative on $\left(2 \theta, \theta+\frac{1}{2}\right)$, and strictly positive on $\left(\theta+\frac{1}{2}, 1+2 \gamma\right)$. So $f_{\theta}+\varphi_{\theta}$ is concave, but not constant, on the semi-circle $\left[\gamma, \gamma+\frac{1}{2}\right]$, and has precisely one local minimum in $[\gamma+1 / 2, \gamma]$. It follows that $f_{\theta}$ satisfies (4) thus completing the proof of Case 1.

Case 2: $\theta \notin[-1 / 8,1 / 8]$.

If we can show that $f_{\theta}$ is Sturmian on some semi-circle $[\gamma, \gamma+1 / 2]$, where $\gamma \in$ $(\theta-1 / 2, \theta)$, it follows from Lemma 6 (ii) that $\theta=\omega(\gamma)$. Write $f=f_{\theta}$, and set $\Delta(x):=(f+\varphi)(x)-(f+\varphi)(x+1 / 2)$, where $\varphi$ is any maximizing function for $f$ (cf. Remark 5 (a)). We wish to show that there exists $\gamma \in(\theta-1 / 2, \theta)$ such that $\Delta$ is strictly positive on $(\gamma, \gamma+1 / 2)$ (and therefore strictly negative on $(\gamma+1 / 2, \gamma)$, since $\Delta(x-1 / 2)=-\Delta(x)$ for all $x \in \mathbb{T})$. For this it suffices to prove that ${ }^{8} \Delta_{+}^{\prime}(x)<0$ for $x \in(\theta, \theta+1 / 2)$, which implies that $\Delta$ is strictly decreasing on $[\theta, \theta+1 / 2]$.

\footnotetext{
${ }^{8}$ Recall that if $g: \mathbb{T} \rightarrow \mathbb{R}$ is Lipschitz then its derivative $g^{\prime}(x)$ at the point $x \in \mathbb{T}$ is defined to be the closed interval

$$
g^{\prime}(x)=\left[g_{-}^{\prime}(x), g_{+}^{\prime}(x)\right]=\bigcap_{\varepsilon>0} \overline{\left\{\frac{g(y)-g(z)}{y-z}: y, z \in(x-\varepsilon, x+\varepsilon), y \neq z\right\}},
$$

which reduces to a singleton (identified with its unique member) when $g$ is differentiable at $x$.
} 
For $x \in \mathbb{T}$, define $\mathcal{H}(x)$ to be the set of sequences $\underline{x}=\left(x_{i}\right)_{i \geq 0}$ such that $x_{0}=x$ and $T\left(x_{i+1}\right)=x_{i}$ for $i \geq 0$, and such that there exists a maximizing function $\varphi$ for $f$ such that

$$
(f+\varphi)\left(x_{i}\right) \geq(f+\varphi)\left(x_{i}+1 / 2\right) \text { for all } i \geq 1 .
$$

Differentiation and iteration of (3) gives that for each $x \in \mathbb{T}$, the interval $\varphi^{\prime}(x)$ is contained in the convex hull of the set

$$
\bigcup_{\underline{x} \in \mathcal{H}(x)} \sum_{i=1}^{\infty} 2^{-i} f^{\prime}\left(x_{i}\right)=\bigcup_{\underline{x} \in \mathcal{H}(x)}\left[\sum_{i=1}^{\infty} 2^{-i} f_{-}^{\prime}\left(x_{i}\right), \sum_{i=1}^{\infty} 2^{-i} f_{+}^{\prime}\left(x_{i}\right)\right] .
$$

It follows that for all $x \in \mathbb{T}$,

$$
\Delta_{+}^{\prime}(x) \leq \sup _{\underline{x} \in \mathcal{H}(x)} \sum_{i=0}^{\infty} 2^{-i} f_{+}^{\prime}\left(x_{i}\right)-\inf _{\underline{y} \in \mathcal{H}(x+1 / 2)} \sum_{i=0}^{\infty} 2^{-i} f_{-}^{\prime}\left(y_{i}\right) .
$$

But if $x \in(\theta, \theta+1 / 2)$ then $f_{-}^{\prime}\left(x_{0}\right)=f^{\prime}(x)=-4$ for any $\underline{x} \in \mathcal{H}(x)$, and $f_{+}^{\prime}\left(y_{0}\right)=$ $f^{\prime}(x+1 / 2)=4$ for any $\underline{y} \in \mathcal{H}(x+1 / 2)$, so (10) becomes

$$
\Delta_{+}^{\prime}(x) \leq-8+\sup _{\underline{x} \in \mathcal{H}(x)} \sum_{i=1}^{\infty} 2^{-i} f_{+}^{\prime}\left(x_{i}\right)-\inf _{\underline{y} \in \mathcal{H}(x+1 / 2)} \sum_{i=1}^{\infty} 2^{-i} f_{-}^{\prime}\left(y_{i}\right) .
$$

Now $\max _{x \in \mathbb{T}}\left(f_{+}^{\prime}(x),-f_{-}^{\prime}(x)\right)=4$, so

$$
\sup _{\underline{x} \in \mathcal{H}(x)} \sum_{i=1}^{\infty} 2^{-i} f_{+}^{\prime}\left(x_{i}\right)-\inf _{\underline{y} \in \mathcal{H}(x+1 / 2)} \sum_{i=1}^{\infty} 2^{-i} f_{-}^{\prime}\left(y_{i}\right) \leq 8 \quad \text { for all } x \in \mathbb{T},
$$

thus (11) implies that $\Delta_{+}^{\prime}(x) \leq 0$ for $x \in(\theta, \theta+1 / 2)$, with equality attained if and only if

$$
\sup _{\underline{x} \in \mathcal{H}(x)} \sum_{i=1}^{\infty} 2^{-i} f_{+}^{\prime}\left(x_{i}\right)=4 \text { and } \inf _{\underline{y} \in \mathcal{H}(x+1 / 2)} \sum_{i=1}^{\infty} 2^{-i} f_{-}^{\prime}\left(y_{i}\right)=-4,
$$

i.e. if and only if

$$
x_{i} \in[\theta-1 / 2, \theta] \quad \text { and } \quad y_{i} \in[\theta, \theta+1 / 2] \quad \text { for all } i \geq 1, \underline{x} \in \mathcal{H}(x), \underline{y} \in \mathcal{H}(x+1 / 2) .
$$

The set of accumulation points of any sequence in $\mathcal{H}(x)$ is a certain compact invariant set, carrying at least one invariant probability measure, which is necessarily $f_{\theta^{-}}$-maximizing. So if (12) holds then the Sturmian measure carried by $[\theta-1 / 2, \theta]$ is $f_{\theta^{-}}$ maximizing, as is the Sturmian measure carried by $[\theta, \theta+1 / 2]$. One of these measures must be the Dirac measure $\delta_{0}$ concentrated at the fixed point 0 , so to show that (12) cannot hold it suffices to prove that $\delta_{0}$ is not $f_{\theta}$-maximizing for $\theta \notin[-1 / 8,1 / 8]$. For this, by symmetry it suffices to consider $\theta \in\left(\frac{1}{8}, \frac{1}{2}\right]$, and find an invariant probability measure whose $f_{\theta}$-average is strictly larger than $f_{\theta}(0)=1-4 \theta$. Indeed this measure may be chosen from the family $\left(m_{n}\right)_{n \geq 2}$ of invariant probability measures supported by the period- $n$ orbit $\left\{1 /\left(2^{n}-1\right), \ldots, 2^{n-1} /\left(2^{n}-1\right)\right\}$, since if $\theta \in U_{1}:=\left[\frac{1}{3}, \frac{1}{2}\right]$ then $\int f_{\theta} d m_{2}=\frac{1}{3}>0>1-4 \theta=f_{\theta}(0)$, if $\theta \in U_{2}:=\left(\frac{1}{4}, \frac{1}{3}\right]$ then $\int f_{\theta} d m_{2}=4 \theta-1>0>$ $1-4 \theta=f_{\theta}(0)$, while if $\theta \in U_{n}:=\left(\frac{2^{n-1}+1}{4\left(2^{n}-1\right)}, \frac{2^{n-2}}{2^{n}-1}\right)$ for $n \geq 3$, then

$$
\int f_{\theta} d m_{n}=1-\frac{4\left(2^{n-1}+1\right)}{n\left(2^{n}-1\right)}-\frac{4(n-4)}{n} \theta>1-4 \theta=f_{\theta}(0) .
$$

Since $\cup_{n \geq 1} U_{n}=(1 / 8,1 / 2]$, the proof is complete. 
If $\varrho(\gamma)$ denotes the rotation number of the Sturmian measure supported by the semi-circle $[\gamma, \gamma+1 / 2]$, then the map $\varrho: \mathbb{T} \rightarrow \mathbb{T}$ is known (see [16]) to be a devil's staircase, i.e. a continuous, weakly increasing degree-1 map such that the preimage of each rational is an interval, and the preimage of each irrational is a single point. Defining $r: \mathbb{T} \rightarrow \mathbb{T}$ by $r:=\varrho \circ \omega^{-1}$, Theorem 7 says that the unique $f_{\theta}$-maximizing measure is the Sturmian measure $S_{\varrho\left(\omega^{-1}(\theta)\right)}=S_{r(\theta)}$. The properties of $\varrho$ and $\omega^{-1}$ imply that $r: \mathbb{T} \rightarrow \mathbb{T}$ is itself a devil's staircase (see Figure 2), so in particular we have:

COROllary 8. Every Sturmian measure is the maximizing measure for at least one member of the piecewise-linear cosine family $\left(f_{\theta}\right)_{\theta \in \mathbb{T}}$. If $\varrho \notin \mathbb{Q}$ then $S_{\varrho}$ is the maximizing measure for precisely one member of $\left(f_{\theta}\right)_{\theta \in \mathbb{T}}$. If $\varrho \in \mathbb{Q}$ then there is a closed interval $\Theta(\varrho)$, with nonempty interior, such that $S_{\varrho}$ is the $f_{\theta}$-maximizing measure for every $\theta \in \Theta(\varrho)$.

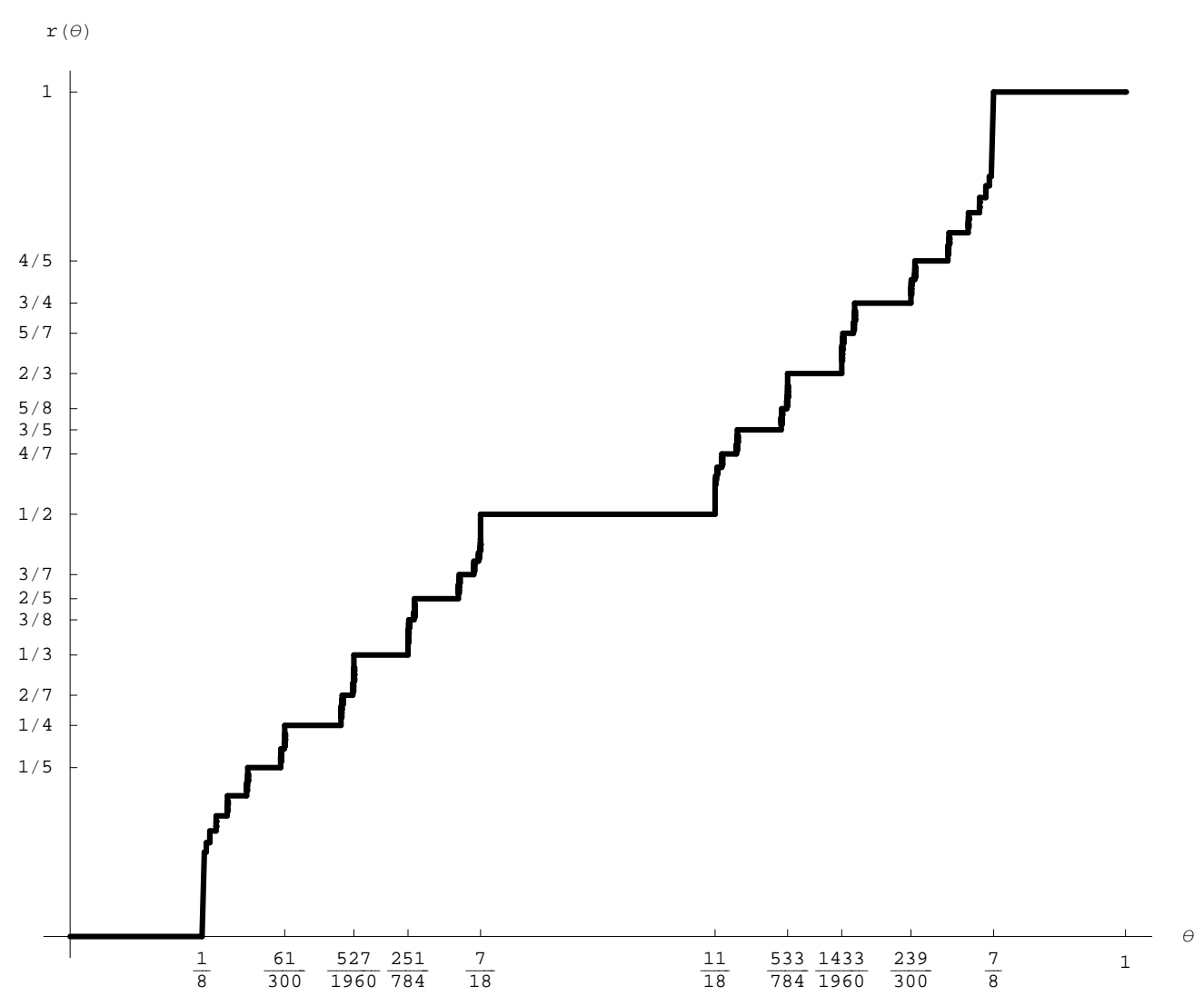

Figure 2. The function $\theta \mapsto r(\theta)$, where $r(\theta)$ denotes the rotation number of the (Sturmian) maximizing measure for $f_{\theta}$.

For rational $\varrho \in \mathbb{T}$, the endpoints of any interval $r^{-1}(\varrho)=\Theta(\varrho)=\left[\theta_{\min }(\varrho), \theta_{\max }(\varrho)\right]$ can be given explicitly, using explicit information about the functions $\omega: \mathbb{T} \rightarrow \mathbb{T}$ (see $[4])$ and $\varrho: \mathbb{T} \rightarrow \mathbb{T}$ (see $[\mathbf{1 6}]$ ). Some of the explicit values for $\theta_{\min }(\varrho)$ and $\theta_{\max }(\varrho)$ are listed in Figure 3 below.

REMARK 9.

(a) Note that the explicit formulae for the intervals $\Theta(p / q)$ means we have precisely identified the Sturmian maximizing measure for (Lebesgue almost) every parameter value $\theta$, to an extent which is not possible for the cosine family $g_{\theta}(x)=\cos 2 \pi(x-\theta)$. 


\begin{tabular}{|c|c|c|}
\hline$\varrho$ & $\theta_{\min }(\varrho)$ & $\theta_{\max }(\varrho)$ \\
\hline \hline 0 & $-1 / 8=-0.125$ & $1 / 8=0.125$ \\
$1 / 6$ & $263 / 1764 \approx 0.149092$ & $295 / 1764 \approx 0.167233$ \\
$1 / 5$ & $647 / 3844 \approx 0.16831$ & $767 / 3844 \approx 0.199541$ \\
$2 / 9$ & $208583 / 1044484 \approx 0.19969$ & $212039 / 1044484 \approx 0.203008$ \\
$1 / 4$ & $61 / 300 \approx 0.20333$ & $77 / 300 \approx 0.25666$ \\
$3 / 11$ & $4303511 / 16760836 \approx 0.25675$ & $4320407 / 16760836 \approx 0.257768$ \\
$2 / 7$ & $16631 / 64516 \approx 0.257781$ & $3317209 / 12387072 \approx 0.267796$ \\
$3 / 10$ & $1494731 / 5581488 \approx 0.267802$ & $90709 / 337590 \approx 0.268696$ \\
$1 / 3$ & $527 / 1960 \approx 0.268878$ & $251 / 784 \approx 0.320153$ \\
$4 / 11$ & $10736023 / 33521672 \approx 0.320271$ & $10757143 / 33521672 \approx 0.3209011$ \\
$3 / 8$ & $11129 / 34680 \approx 0.320905$ & $42371 / 130050 \approx 0.325805$ \\
$5 / 13$ & $43718531 / 134184962 \approx 0.325808$ & $43771779 / 134184962 \approx 0.326205$ \\
$5 / 12$ & $4112657 / 11179350 \approx 0.36788$ & $4120849 / 11179350 \approx 0.368613$ \\
$3 / 7$ & $11891 / 32258 \approx 0.36862$ & $12339 / 32258 \approx 0.38251$ \\
$4 / 9$ & $199795 / 522242 \approx 0.382571$ & $202099 / 522242 \approx 0.386983$ \\
$1 / 2$ & $7 / 18 \approx 0.388888$ & $11 / 18 \approx 0.611111$ \\
\hline
\end{tabular}

FiguRE 3. Endpoints of intervals $r^{-1}(p / q)=\left[\theta_{\min }(p / q), \theta_{\max }(p / q)\right]$ for certain $p / q \in[0,1 / 2]$. Certain other values (for $p / q \in(1 / 2,1)$ ) follow from the identity $r(x+1 / 2)=r(x-1 / 2)$.

In $[4]$ we also note that, for sufficiently large $q$, the interval lengths $\theta_{\max }(p / q)-\theta_{\min }(p / q)$ are equal to $K q 2^{q-1} /\left(2^{q}-1\right)^{2}$ for one of five explicit constants $K$; asymptotically this agrees with the lengths of the corresponding parameter intervals for the family $g_{\theta}$ (see $[14,24])$.

(b) As we have seen, the proof of Theorem 7 (and therefore Theorem 1) is informed by some theoretical concepts introduced by Bousch in [7], notably the Sturmian and pre-Sturmian conditions. One difference in the approach used here is that the proof revolves principally around the pre-Sturmian, rather than the Sturmian, condition. A further difference is that our proof is entirely self-contained, not relying on numerical approximations such as those required in [7] for the cosine family.

\section{The maximum ergodic average}

Now we would like to understand the variation with $\theta$ of the maximum ergodic average

$$
\alpha(\theta):=\max _{\mu \in \mathcal{M}} \int f_{\theta} d \mu
$$

of the piecewise-linear cosine function $f_{\theta}$. This function $\alpha: \mathbb{T} \rightarrow \mathbb{R}$ turns out to be countably piecewise affine, with unique global maximum at $\theta=0$, and global minimum at $\theta \in[7 / 18,11 / 18]$ (when $S_{1 / 2}=\left(\delta_{1 / 3}+\delta_{2 / 3}\right) / 2$ is $f_{\theta}$-maximizing). It is piecewise monotone (but not piecewise affine), and turns out to have precisely 23 intervals of monotonicity (see Theorem 11); the endpoints of these intervals of monotonicity can be identified explicitly, and turn out to be either endpoints of some 
$\Theta(p / q)=r^{-1}(p / q)$ (cf. Figure 3), or certain exceptional values ${ }^{9} \theta_{\infty}(p / q)$ for $p / q=$ $0, \pm 2 / 7, \pm 3 / 10, \pm 1 / 3, \pm 3 / 8$. The function $\alpha$ is shown in Figure 4 , though some of its intervals of monotonicity are too small to be visible here; the smallest such interval has length $4096 / 68736249855 \approx 6 \times 10^{-8}$.

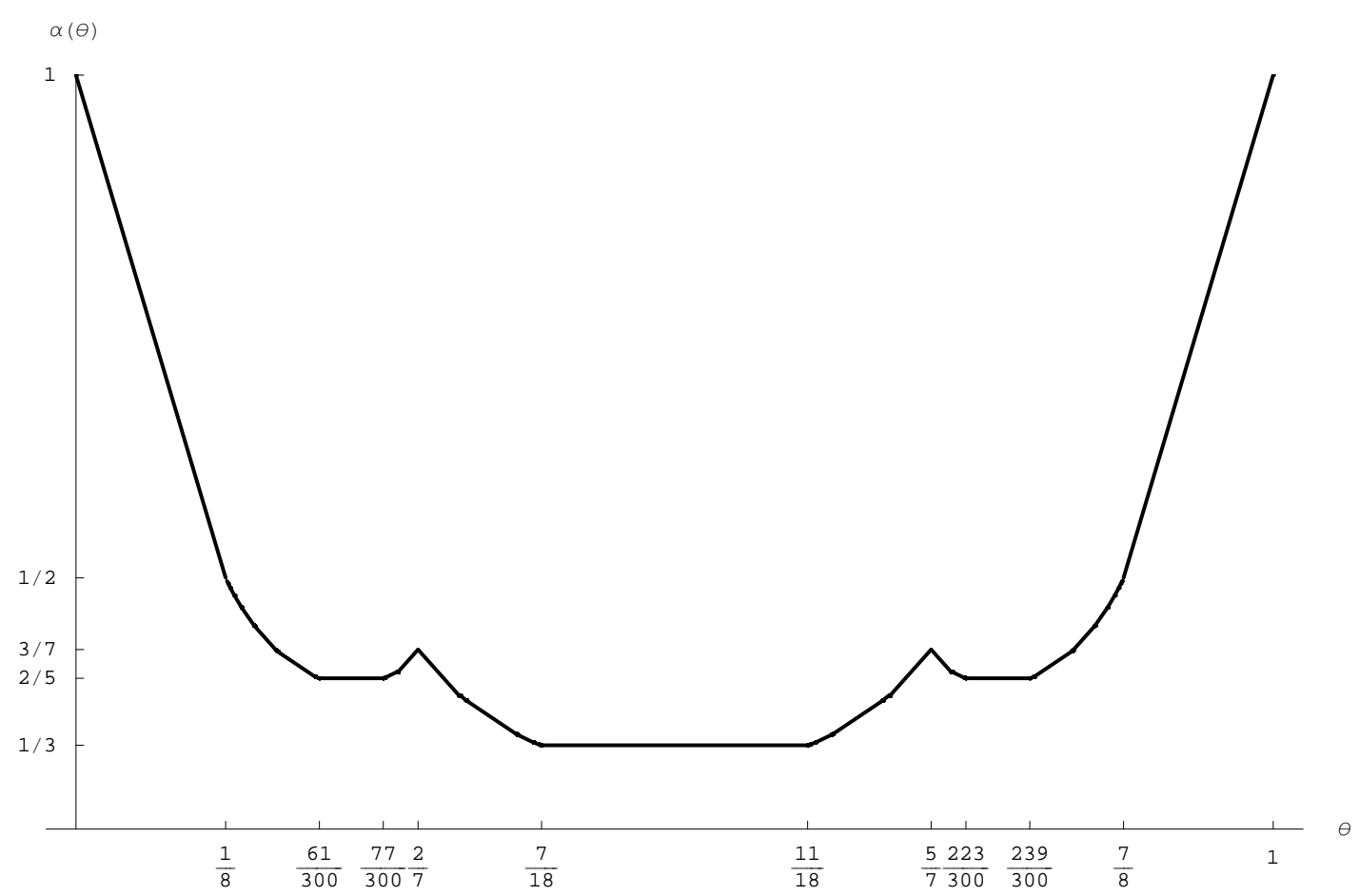

Figure 4. Graph of the countably piecewise affine function $\alpha(\theta):=$ $\max _{\mu \in \mathcal{M}} \int f_{\theta} d \mu$. Only 9 of its 23 intervals of monotonicity are visible.

To prove the above facts about $\alpha$ we first require the following result from [3]:

Lemma 10. [3, Cor. 6.6] (cf. also [4, Thm. 2.8]) Suppose $\varrho(\gamma)$ is the rational $p / q$ (where $q \in \mathbb{N}$ and the integer $0<p<q$ is coprime to $q$ ), and $J_{i}:=\left[s_{i}, s_{i+1}\right]$ for $1 \leq i \leq q-1$, where $s_{1}<\ldots<s_{q}$ denotes the Sturmian orbit of rotation number $p / q$. Then

$$
\omega(\gamma) \in \begin{cases}J_{q-2 p} & \text { if } \varrho(\gamma) \in(0,2 / 7) \\ J_{4 q-12 p} & \text { if } \varrho(\gamma) \in(2 / 7,3 / 10) \\ J_{2 q-5 p} & \text { if } \varrho(\gamma) \in(3 / 10,1 / 3) \\ J_{2 q-4 p} & \text { if } \varrho(\gamma) \in(1 / 3,3 / 8) \\ J_{q-p} & \text { if } \varrho(\gamma) \in(3 / 8,1 / 2] .\end{cases}
$$

THEOREM 11. The maximum ergodic average function $\alpha: \mathbb{T} \rightarrow \mathbb{R}$, defined by $\alpha(\theta)=\max _{\mu \in \mathcal{M}} \int f_{\theta} d \mu$, is even and countably piecewise affine. Its maximum value is 1 , attained uniquely at $\theta=0$, and its minimum value is $1 / 3$, attained uniquely on the interval $[7 / 18,11 / 18]$. The function $\alpha$ is piecewise monotone, with precisely 23 monotonicity intervals ${ }^{10}$. Those endpoints of monotonicity intervals lying between 0

\footnotetext{
${ }^{9}$ These exceptional values $\theta_{\infty}(p / q)$ are denoted $\omega_{\infty}(p / q)$ in $[\mathbf{3}]$, where the significance of the nine exceptional rotation numbers $0, \pm 2 / 7, \pm 3 / 10, \pm 1 / 3, \pm 3 / 8$ is described.

${ }^{10} \mathrm{By}$ a monotonicity interval we mean a locally maximal interval on which $\alpha$ is either strictly increasing, or strictly decreasing, or constant.
} 
and $1 / 2$ are, in increasing order,

$$
\begin{gathered}
\theta_{1}:=\theta_{\min }(1 / 4)=\frac{61}{300} \approx 0.20333, \quad \theta_{2}:=\theta_{\max }(1 / 4)=\frac{77}{300} \approx 0.25666, \\
\theta_{3}:=\theta_{\infty}(2 / 7)=\frac{34}{127} \approx 0.26771, \quad \theta_{4}:=\theta_{\min }(7 / 24)=\frac{147258372059}{549889998840} \approx 0.26779605, \\
\theta_{5}:=\theta_{\max }(7 / 24)=\frac{147258404827}{549889998840} \approx 0.26779611, \quad \theta_{6}:=\theta_{\infty}(3 / 10)=\frac{274}{1023} \approx 0.26783, \\
\theta_{7}:=\theta_{\max }(3 / 10)=\frac{90709}{337590} \approx 0.26869, \quad \theta_{8}:=\theta_{\infty}(1 / 3)=2 / 7 \approx 0.28571, \\
\theta_{9}:=\theta_{\min }(3 / 8)=\frac{11129}{34680} \approx 0.32090, \quad \theta_{10}:=\theta_{\infty}(3 / 8)=\frac{82}{255} \approx 0.32156, \\
\theta_{11}:=\theta_{\min }(1 / 2)=\frac{7}{18} \approx 0.38888,
\end{gathered}
$$

and $\alpha$ is decreasing on $\left[0, \theta_{1}\right]$, constant on $\left[\theta_{1}, \theta_{2}\right]$, increasing on $\left[\theta_{2}, \theta_{3}\right]$, decreasing on $\left[\theta_{3}, \theta_{4}\right]$, constant on $\left[\theta_{4}, \theta_{5}\right]$, increasing on $\left[\theta_{5}, \theta_{6}\right]$, constant on $\left[\theta_{6}, \theta_{7}\right]$, increasing on $\left[\theta_{7}, \theta_{8}\right]$, decreasing on $\left[\theta_{8}, \theta_{9}\right]$, constant on $\left[\theta_{9}, \theta_{10}\right]$, decreasing on $\left[\theta_{10}, \theta_{11}\right]$, and constant on $\left[\theta_{11}, 1 / 2\right]$.

Proof. Since $\{\theta \in \mathbb{T}: r(\theta) \in \mathbb{Q}\}$ is dense in $\mathbb{T}$, and $\alpha$ is clearly (4-Lipschitz) continuous, to prove that $\alpha$ is monotone on any interval it suffices to show that it is monotone on the intersection of this interval with $\{\theta \in \mathbb{T}: r(\theta) \in \mathbb{Q}\}$.

For any $x \in \mathbb{T}$, the map $\theta \mapsto f_{\theta}(x)$ is affine (hence monotone) with slope \pm 4 on any interval $\left[\theta^{-}, \theta^{+}\right]$disjoint from $\{x, x+1 / 2\}$. Thus, if $\theta$ is such that $f_{\theta}$ has periodic maximizing measure, supported by the Sturmian periodic orbit $s_{1}<\ldots<s_{q}$, then the formula $\alpha(\theta)=q^{-1} \sum_{i=1}^{q} f_{\theta}\left(s_{i}\right)$ implies that the local monotonicity of $\alpha$ at $\theta$ is entirely determined by whether most points in the periodic orbit lie to the left, or to the right, of $\theta$. Precisely, $\alpha$ is locally decreasing (respectively increasing) if more than half of the points lie to the left (respectively the right) of $\theta$, and is locally constant if exactly half of the points lie on either side of $\theta$ (in this last case the orbit is necessarily of even period).

If $\theta \in\left(0, \theta_{\max }(0)\right]=(0,1 / 8]$ then the fixed point 0 is maximizing, and is to the left of $\theta$, so $\alpha$ is decreasing on $(0,1 / 8]$.

Now suppose that $\theta$ is such that the $f_{\theta}$-maximizing measure is $S_{p / q}$ for $0<p / q<$ $2 / 7$. Lemma 10 implies that $\theta$ lies in the interior of $J_{q-2 p}$, so precisely $q-2 p$ of the $q$ points in $\mathcal{O}_{p / q}$ lie to the left of $\theta$. So if $0<p / q<1 / 4$ then most points in the orbit lie to the left of $\theta$, hence $\alpha$ is locally decreasing at $\theta$, if $p / q=1 / 4$ then exactly two points in the orbit $\{1 / 15,2 / 15,4 / 15,8 / 15\}$ lie to either side of $\theta$, so $\alpha$ is locally constant, while if $1 / 4<p / q<2 / 7$ then most points lie to the right of $\theta$, hence $\alpha$ is locally increasing at $\theta$. It follows that $\alpha$ is decreasing on $\left[\theta_{\max }(0), \theta_{\min }(1 / 4)\right]$, constant on $\left[\theta_{\min }(1 / 4), \theta_{\max }(1 / 4)\right]$, and increasing on $\left[\theta_{\max }(1 / 4), \theta_{\min }(2 / 7)\right]$.

Now suppose that the $f_{\theta}$-maximizing measure is $S_{2 / 7}$. The point $\theta_{\infty}(2 / 7)=34 / 127$ is the central point in $\mathcal{O}_{2 / 7}=\{9 / 127,17 / 127,18 / 127,34 / 127,36 / 127,68 / 127,72 / 127\}$. Therefore, if $\theta \in\left[\theta_{\min }(2 / 7), \theta_{\infty}(2 / 7)\right)$ then three points in the orbit lie to the left of $\theta$, so $\alpha$ is locally increasing at $\theta$, while if $\theta \in\left(\theta_{\infty}(2 / 7), \theta_{\max }(2 / 7)\right]$ then four points in the orbit lie to the left of $\theta$, so $\alpha$ is locally decreasing at $\theta$.

Now suppose that $\theta$ is such that the $f_{\theta}$-maximizing measure is $S_{p / q}$ for $2 / 7<p / q<$ $3 / 10$. By Lemma 10, $\theta$ lies in the interior of $J_{4 q-12 p}$, so precisely $4 q-12 p$ of the $q$ 
points in $\mathcal{O}_{p / q}$ lie to the left of $\theta$. Now $4 q-12 p>q / 2$ if and only if $p / q<7 / 24$, so $\alpha$ is decreasing on $\left[\theta_{\max }(2 / 7), \theta_{\min }(7 / 24)\right]$; similarly, $\alpha$ is constant on $\left[\theta_{\min }(7 / 24), \theta_{\max }(7 / 24)\right]$ and increasing on $\left[\theta_{\max }(7 / 24), \theta_{\min }(3 / 10)\right]$.

By now the general argument is clear; to complete the proof we may continue as above, examining separately the remaining exceptional rotation numbers $(3 / 10,1 / 3$, and $3 / 8$ ), and the rationals between them (for which we use Lemma 10 to locate $\theta$ relative to the partition $\left\{J_{i}\right\}$, then argue as above).

\section{References}

[1] J.-P. Allouche \& J. Shallit, Automatic sequences. Theory, applications, generalizations, Cambridge University Press, Cambridge, 2003.

[2] V. Anagnostopoulou \& O. Jenkinson, Which beta-shifts have a largest invariant measure? Jour. London Math. Soc., 79 (2009), 445-464.

[3] V. Anagnostopoulou, K. Díaz-Ordaz, O. Jenkinson \& C. Richard, Entrance time functions for flat spot maps, Nonlinearity, 23 (2010), 1477-1494.

[4] V. Anagnostopoulou, K. Díaz-Ordaz, O. Jenkinson \& C. Richard, The flat spot standard family: variation of the entrance time median, preprint, 2010.

[5] J. Berstel \& P. Séébold, Sturmian words, in: M. Lothaire, Algebraic combinatorics on words, Encyclopaedia of mathematics and its applications, vol. 90, Cambridge University Press, 2002, pp. $45-110$.

[6] A. Baraviera, R. Leplaideur \& A. Lopes, Selection of measures for a potential with two maxima at the zero temperature limit, preprint, 2010.

[7] T. Bousch, Le poisson n'a pas d'arêtes, Ann. Inst. Henri Poincaré (Proba. et Stat.), 36 (2000), 489-508.

[8] T. Bousch, La condition de Walters, Ann. Sci. ENS, 34, (2001), 287-311.

[9] T. Bousch, Nouvelle preuve d'un théorème de Yuan et Hunt, Bull. Soc. Math. France, 126 (2008), 227-242.

[10] T. Bousch \& O. Jenkinson, Cohomology classes of dynamically non-negative $C^{k}$ functions, Invent. Math., 148 (2002), 207-217.

[11] F. Branco, Sub-actions and maximizing measures for one-dimensional transformations with a critical point, Discrete $\&$ Cont. Dyn. Sys., 17 (2007), 271-280.

[12] J. Brémont, On the behaviour of Gibbs measures at temperature zero, Nonlinearity 16 (2003), 419-426.

[13] J. Brémont, Entropy and maximizing measures of generic continuous functions, C. R. Math. Acad. Sci. Série I, 346 (2008), 199-201.

[14] J. Brémont \& S. Seuret, The singularity spectrum of the fish's boundary, Ergod. Th. EG Dyn. Sys., 28 (2008), 49-66.

[15] X. Bressaud \& A. Quas, Rate of approximation of minimizing measures, Nonlinearity, 20 (2007), 845-853.

[16] S. Bullett \& P. Sentenac, Ordered orbits of the shift, square roots, and the devil's staircase, Math. Proc. Camb. Phil. Soc., 115 (1994), 451-481.

[17] J.-R. Chazottes \& M. Hochman, On the zero-temperature limit of Gibbs states, Comm. Math. Phys., to appear.

[18] D. Collier \& I. D. Morris, Ergod. Th. \& Dyn. Sys., 28 (2008), 1081-1090.

[19] G. Contreras, A. Lopes, \& Ph. Thieullen, Lyapunov minimizing measures for expanding maps of the circle, Ergod. Th. ES Dyn. Sys., 21 (2001), 1379-1409.

[20] J.-P. Conze \& Y. Guivarc'h, Croissance des sommes ergodiques et principe variationnel, manuscript, circa 1993.

[21] A. Fathi, Weak KAM theorem in Lagrangian dynamics, Cambridge Studies in Advanced Mathematics, No. 88, Cambridge University Press, to appear.

[22] J-M. Gambaudo, O. Lanford III, \& C. Tresser, Dynamique symbolique des rotations, C. $R$. Acad. Sc. Paris, 299 (1984), 823-826.

[23] E. Garibaldi, A. O. Lopes \& Ph. Thieullen, On calibrated and separating sub-actions, Bull. Braz. Math. Soc., 40 (2009), 577-602. 
[24] B. R. Hunt and E. Ott, Optimal periodic orbits of chaotic systems occur at low period, Phys. Rev. E, 54 (1996), 328-337.

[25] O. Jenkinson, Conjugacy rigidity, cohomological triviality, and barycentres of invariant measures, Ph. D. thesis, University of Warwick, 1996.

[26] O. Jenkinson, Frequency locking on the boundary of the barycentre set, Experimental Mathematics, 9 (2000), 309-317.

[27] O. Jenkinson, Geometric barycentres of invariant measures for circle maps, Ergod. Th. ES Dyn. Sys., 21 (2001), 511-532.

[28] O. Jenkinson, Rotation, entropy, and equilibrium states, Trans. Amer. Math. Soc., 353 (2001), 3713-3739.

[29] O. Jenkinson, Ergodic optimization, Discrete \& Cont. Dyn. Sys., 15 (2006), 197-224.

[30] O. Jenkinson, Every ergodic measure is uniquely maximizing, Discrete \& Cont. Dyn. Sys., 16 (2006), 383-392.

[31] O. Jenkinson, Optimization and majorization of invariant measures, Electron. Res. Announc. Amer. Math. Soc., 13 (2007), 1-12.

[32] O. Jenkinson, A partial order on ×2-invariant measures, Math. Res. Lett., 15 (2008), 893-900.

[33] O. Jenkinson, R. D. Mauldin \& M. Urbański, Zero temperature limits of Gibbs-equilibrium states for countable alphabet subshifts of finite type, J. Stat. Phys., 119 (2005), 765-776.

[34] O. Jenkinson \& J. Steel, Majorization of invariant measures for orientation-reversing maps, Ergod. Th. \& Dyn. Sys., 30 (2010), to appear.

[35] R. Leplaideur, A dynamical proof for the convergence of Gibbs measures at temperature zero, Nonlinearity, 18 (2005), 2847-2880.

[36] A. O. Lopes \& Ph. Thieullen, Sub-actions for Anosov diffeomorphisms, Geometric methods in dynamics II. Astérisque vol. 287, 2003

[37] A. O. Lopes \& Ph. Thieullen, Sub-actions for Anosov flows, Ergod. Th. \& Dyn. Sys., 25 (2005), $605-628$

[38] I. D. Morris, Entropy for zero-temperature limits of Gibbs-equilibrium states for countablealphabet subshifts of finite type, J. Stat. Phys., 126 (2007), 315-324.

[39] I. D. Morris, A sufficient condition for the subordination principle in ergodic optimization, Bull. London Math. Soc., 39 (2007), 214-220.

[40] I. D. Morris, Maximizing measures of generic Hölder functions have zero entropy, Nonlinearity, 21 (2008), 993-1000.

[41] I. D. Morris, The Mañé-Conze-Guivarc'h lemma for intermittent maps of the circle, Ergod. Th. \& Dyn. Sys., 29 (2009), 1603-1611.

[42] I. D. Morris, Ergodic optimization for generic continuous functions, Discrete 6 Cont. Dyn. Sys., 27 (2010), 383-388.

[43] M. Morse \& G. A. Hedlund, Symbolic Dynamics II. Sturmian Trajectories, Amer. J. Math., 62 (1940), 1-42.

[44] M. Pollicott \& R. Sharp, Livsic theorems, maximizing measures and the stable norm, Dyn. Syst., 19 (2004), 75-88.

[45] N. Pytheas Fogg, Substitutions in dynamics, arithmetics and combinatorics, Springer Lecture Notes in Mathematics vol. 1794, 2002.

[46] S. V. Savchenko, Homological inequalities for finite topological Markov chains, Funct. Anal. Appl., 33 (1999), 236-238.

[47] R. Souza, Sub-actions for weakly hyperbolic one-dimensional systems, Dyn. Syst., 18 (2003), 165-179.

[48] F. A. Tal \& S. Addas-Zanata, On maximizing measures of homeomorphisms on compact manifolds, Fund. Math., 200 (2008), 145-159.

[49] F. A. Tal \& S. Addas-Zanata, Maximizing measures for endomorphisms of the circle, Nonlinearity, 21 (2008), 2347-2359.

[50] P. Veerman, Symbolic dynamics of order-preserving orbits, Physica D, 29 (1987), 191-201.

[51] G. Yuan \& B. R. Hunt, Optimal orbits of hyperbolic systems, Nonlinearity, 12 (1999), 12071224. 
Vasso Anagnostopoulou; School of Mathematical Sciences, Queen Mary, UniverSity of London, Mile End RoAd, London, E1 4NS, UK.

vaa@maths.qmul.ac.uk

Karla Díaz-Ordaz; School of Mathematical Sciences, Queen Mary, University of London, Mile End RoAd, London, E1 4NS, UK.

karla@maths.qmul.ac.uk

Oliver Jenkinson; School of Mathematical Sciences, Queen Mary, University of London, Mile End Road, London, E1 4NS, UK.

omj@maths . qmul . ac.uk

www . maths. qmul. ac.uk/ omj

Catherine Richard; School of Mathematical Sciences, Queen Mary, University of London, Mile End RoAd, London, E1 4NS, UK.

csr@maths.qmul .ac.uk 